\title{
The influence of hubs in the structure of a neuronal network during an epileptic seizure
}

\author{
Abner Cardoso Rodrigues ${ }^{1,3}$, Hilda A. Cerdeira ${ }^{2}$, and Birajara Soares Machado Ma $^{3, a}$ \\ 1 Bioinformatics Interunits Graduate Program, University of São Paulo, São Paulo, Brazil \\ 2 Instituto de Física Teórica, Universidade Estadual Paulista, São Paulo, Brazil \\ ${ }^{3}$ Hospital Israelita Albert Einstein, São Paulo, Brazil
}

Received 6 October 2015 / Received in final form 14 January 2016

Published online 29 February 2016

\begin{abstract}
In this work, we propose changes in the structure of a neuronal network with the intention to provoke strong synchronization to simulate episodes of epileptic seizure. Starting with a network of Izhikevich neurons we slowly increase the number of connections in selected nodes in a controlled way, to produce (or not) hubs. We study how these structures alter the synchronization on the spike firings interval, on individual neurons as well as on mean values, as a function of the concentration of connections for random and non-random (hubs) distribution. We also analyze how the post-ictal signal varies for the different distributions. We conclude that a network with hubs is more appropriate to represent an epileptic state.
\end{abstract}

\section{Introduction}

Anomalies in the connectome, the set of all neurons and brain connections, are present in patients with neurological disorders which affect a large number of subjects worldwide [1]. For example, reduced frontal hub connectivity was reported in patients with schizophrenia [2,3], abnormal modular connectivity was identified in children with autism [4], network hubs also plays an important role in the network from patients with Alzheimer's disease [5-7] and epilepsy [8].

Despite these findings of abnormal network patterns in patients with brain dysfunctions, the way which connectome modulates the brain function, and consequently, the impact of an anomalous network topology in the brain dynamics, is still poorly understood. Theoretical results reveal the influence of the network on dynamical processes [9], such as the importance of the node degree on cascading behaviors [10], the role of the links to opinion spreading in social graphs [11] and the degree distribution effect in percolation phenomena [12].

In this work, we investigate the effect of the network topology in a remarkable emergent property of the brain dynamics: the synchronization. Synchronized activity occurs during healthy brain states such as sleep but also in unhealthy states, like during epileptic seizures [13]. In an episode of seizure (also called ictal period), the

a e-mail: birasm@gmail.com 
brain activity is highly synchronized (hyper-synchronism), showing a very similar eletrophisiological pattern between almost all cortical regions.

Some well documented patterns found in functional networks during seizures are the presence of hubs, the strenghtening of the coupling between brain signals, concave like evolution of the clustering coefficient and characteristic path length along time [14]. An interesting question is how these patterns are related with the hypersynchronization found during the ictal period. Has the network topology a causal effect over the synchronization? How is the interrelation of structure with the dynamics of propagation and the ending of the seizure?

In order to investigate the role of the networks over the hypersynchronization feature of the seizure,we simulated a neuronal network with hubs, a topology that has been shown in [15] to appear during the ictal period. Some analytical and simulation studies point to a dependence between synchronizability of a network and the presence of highly connected nodes [16-18], while other works points towards the opposite direction [19].

Moreover, as these epileptic networks show a time dependent topology, with two clearly distincts states of ictal and non-ictal activity, we analyzed the influence of the ictal network to the post-ictal signal, when the healthy functional structure is restored. In this way, we can observe how a topological configuration can affect the signal's properties.

These results can be valuable to a new interpretation of this time-varying brain topology but also for artificial systems where synchronization is an important feature, as computer networks or brain inspired models.

\section{Materials and methods}

Our simulations employ the Izhikevich neuron model, known for its simplicity and efficiency in modelling neurons and for simulating a great range of signals emitted by real neurons. It is described by the following equations:

$$
\begin{aligned}
& \frac{d v}{d t}=0.04 v^{2}+5 v+140-u+I \\
& \frac{d u}{d t}=a(b v-u)
\end{aligned}
$$

with the after-spike resetting rule in the case of $v \geq 30 \mathrm{mV}$ :

$$
\begin{aligned}
& v \leftarrow c \\
& u \leftarrow u+d,
\end{aligned}
$$

where $v$ is the membrane potential, $u$ is the membrane recovery rate and $I$ represents the input from the thalamus. The set of parameters $a, b, c$ and $d$ are the same as in Ref. [20]. Our simulations were integrated using the Euler method with a step of 0.05 . All networks were created with 1000 neurons with the excitatory-inhibitory ratio fixed in 4:1, the connections are weighted and directed. The network starts with $30 \%$ of the total links initialized, more connections are added in random nodes to simulate the presence of hubs.

These connections were added gradually, governed by two parameters: the percentage of nodes that will receive more connections and will became hubs (hub ratio, HR) and the percentage of connections added per node which in all simulations was fixed at $70 \%$. For example, hubs ratio of 0.1 means that $10 \%$ of the total number of nodes will be linked with $70 \%$ of others nodes. To compare the effect, the same amount of 
connections added to create the network with hubs is added in a random fashion in another network and the behaviour of spiking of both networks were recorded, using the same random parameters of the neurons in both.

The simulations were analyzed considering the discrete events of the firing of each neuron along time and the sum of potential from each neuron, creating a local field potential (LFP) for the entire network. Considering only the firings we measured the synchrony employing the spike distance (SD) method [21], which is inversely proportional to the level of synchronism among them.

We employed two measures to characterize the LFP: the Lempel-Ziv complexity $C_{\mathrm{LZ}}$ and the Hurst exponent $H$. While the Lyapunov exponent measures the intensity of chaotic behavior determination, the complexity gives a probable interpretation for a biased-random degree. In some cases the Lempel-Ziv approach may be an efficient alternative to analyze dynamical behaviors in time series. Also, the method does not require embedding and the series can be interpreted as a binary signal generated by a source. More details and examples see [22].

The Hurst exponent is a measure of long term memory (similar to autocorrelation) and fractality [23]. If the exponent $H>0.5$, the time series is called persistent, as an increase in the present is probably followed by a later increase, whereas $H<0.5$ indicates an antipersistent regime i.e. an increase in the present are more likely to be followed by a lower value [24]. We employed this measure to characterize the LFP after the removal of network hubs, in a way to see the impact os such structure in the signal.

\section{Results and discussion}

In this section we propose a model, and the corresponding simulations, in which by modifying the topology of the network in two different ways: increasing the connectivity in a random way and increasing it in a controlled way, augmenting the number of hubs, we obtain the following: the spike activity of a single neuron is more regular by increasing the number of hubs; the complexity of the signal decreases with the number of hubs and for some miliseconds after the seizure; the post ictal signal retains some of the properties of the crisis.

\section{Enhanced synchronization in Neuronal networks with hubs}

Our first simulation aims to show the influence of hubs in the synchronization among neurons. Figure 1 shows the effect of the hubs on the individual spikes of single neurons and on the LFP signal. The upper part of the Figs. 1a and 1b represent the LFP for 0.3 and 0.7 hubs ratio respectively. Note the regularity of the signal with increasing number of hubs. The bottom frame shows the individual firing patterns along time, evidencing the local synchronism which results in the LFP shown above. In this case, almost all neurons were firing at the same time and the network behaves as a single oscillator.

For each value of $\mathrm{HR}$, from 0.1 to $0.9,100$ networks were created using the random and non-random procedure, then the mean of the SD over the 100 networks are calculated. These results are summarized in Table 1 . The averaged spike distance for the networks with hubs $\left(S D_{\mathrm{NR}}\right)$ shows a similar level of synchronization when compared to the nerworks with random connections $\left(S D_{\mathrm{R}}\right)$ until hubs ratio 0.3 , after this point, the synchronization of the non-random networks converges more strongly as more hubs were added. 
(a)
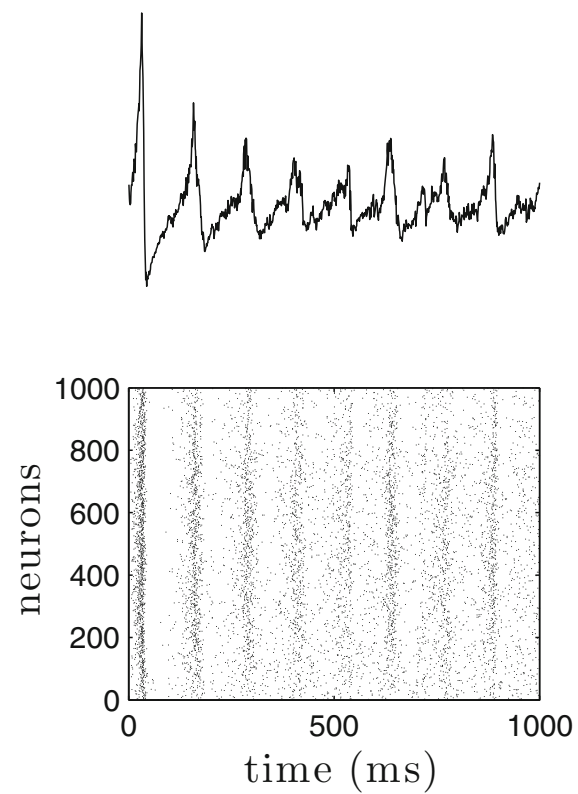

(b)
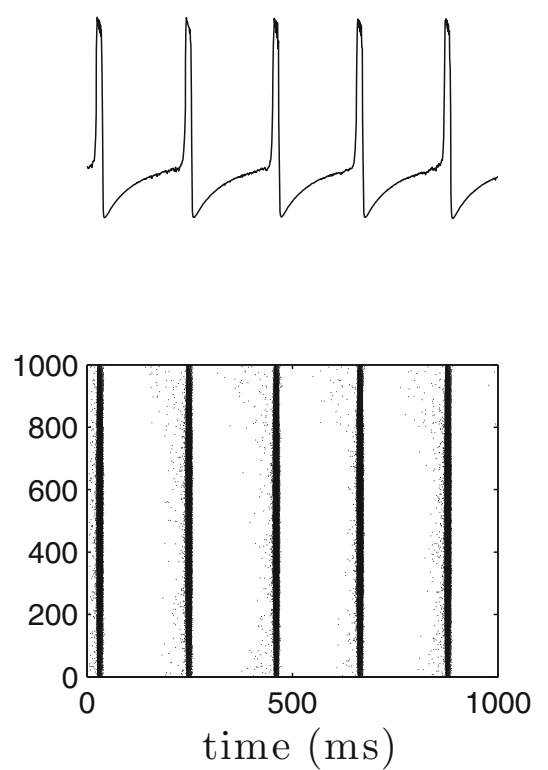

Fig. 1. An example of LFP and spike firings of two networks with hubs ratio 0.3 (left) and 0.7 (right).

The creation of the hubs caused a great impact on $S D_{\mathrm{NR}}$ for values 0.4 and 0.5 , but after that, the decline in the value of SD remained constant but slower. This may be an indication of the existence of an upper bound on network synchronization using the strategy of adding non-random connections [25].

The $S D_{\mathrm{R}}$ stays around 0.2 until HR 0.8 and 0.9 , when it shows a level of synchronization similar to $S D_{\mathrm{NR}}$, but this similarity is possibly due a high number of connections. That is, when the number of connections is very large, the presence of hubs is diluted and both random and non-random configurations are equivalent.

The gain column in the Table 1 is defined as $1-S D_{\mathrm{R}} / S D_{\mathrm{NR}}$, it is a simple ratio to compare the impact of the addition of hubs over random connections. The peak of this measure was between the interval 0.5 to 0.8 . After 0.8 there is a drop in this ratio, reflecting the gain of efficiency of random connections, due to a high density of connections.

With regard to focal epilepsy, a previous work shows that only $35 \%$ of hubs nodes found during the seizure are related to the seizure onset zone (SOZ), i.e. the region responsible for the seizure onset and propagation [26]. This suggests that other regions but the SOZ may have an important role in the seizure evolution $[26,27]$. The results presented here point out to the importance of these hub nodes on the emergence of synchronization in neuronal networks. However, little is known about the mechanism behind the emergence of these abnormal hubs during the seizures. How the brain networks switches from a healthy to an unhealthy state when the number of hubs increases? In addition, this simulation presented a way to generate a highly synchronized pattern, as found during seizures, without modifying the parameters of the neurons, only the connections between them. This is a more realitic approach to model seizures since epilepsy is probably a consequence of anomalous brain connectivity $[13]$. 
Table 1. Spike distance measure for networks with random $\left(S D_{\mathrm{R}}\right)$ and non-random $\left(S D_{\mathrm{NR}}\right)$ connections. The third column (gain) is calculated as $1-S D_{\mathrm{R}} / S D_{\mathrm{NR}}$.

\begin{tabular}{c|ccc} 
hubs ratio & $S D_{\mathrm{R}}$ & $S D_{\mathrm{NR}}$ & gain \\
\hline \hline & & & \\
0.1 & 0.267 & 0.267 & 0 \\
0.2 & 0.266 & 0.263 & 0.011 \\
0.3 & 0.264 & 0.230 & 0.128 \\
0.4 & 0.259 & 0.105 & 0.594 \\
0.5 & 0.253 & 0.048 & 0.810 \\
0.6 & 0.239 & 0.033 & 0.861 \\
0.7 & 0.210 & 0.029 & 0.861 \\
0.8 & 0.143 & 0.028 & 0.804 \\
0.9 & 0.064 & 0.027 & 0.578 \\
\hline \hline
\end{tabular}

\section{Organized networks have a less complex LFP}

In addition to the analysis of the neuronal firings, we estimated the Lempel-Ziv complexity $C_{\mathrm{LZ}}[28]$ over the LFP from two networks: a network with hubs and the network with randomly distributed connections. In order to calculate the $C_{\mathrm{LZ}}, 100$ networks were created for both configurations. In this way, we have a distribution of $C_{\mathrm{Lz}}$ and then we compared the medians of these using a statistical test.

Figure $2 \mathrm{a}$ shows the difference of the median of the $C_{\mathrm{LZ}}$ for the LFP of networks with random connections $\left(\mathrm{N}_{R}\right)$ and nonrandom $\left(\mathrm{N}_{\mathrm{NR}}\right)$ as a function of the HR. The positive trend is well defined from 0.4 until 0.8 when it stabilizes. In this interval, the neurons from nonrandom network were more synchronized and thus, the LFP is less complex than the random network. Notice that the conclusion about the network synchronization similar to the one obtained with the SD measure (see Table 1), since for HR greater than 0.8 there is no increase in the difference of complexity.

This result is in accordance with the one presented by Stam [29], who argues that the seizure may be related with the loss of complexity of the signal since we also found less complexity when the network is more synchronized. Furthermore, this measure can give an idea about the structure of the network and the generator mechanism of the LFP signal: a more organized structure leads to high synchronization and less complexity.

At the bottom part of the Fig. 2 we show the $p$-value for the Wilcoxon test performed over the two $C_{\mathrm{LZ}}$ distributions used in the upper figure. The difference between the two sets was significative from HR 0.4, indicating that from this point the possible values of $C_{\mathrm{LZ}}$ calculated from the LFP were too different. The level of significance used here was $\alpha=0.01$.

\section{Impact of the structure during the ictal period on the signal of the post-ictal period}

Functional brain networks exhibit a very dynamic structure since it is constantly evolving as different cognitive tasks are performed. This evolving structure is also present during seizures, for example the centrality measures of nodes shows a temporal evolution [30]. An important question to the understanding of epilepsy is how the network during the ictal period affects the post-ictal or even the effect of these networks to the normal behavior between the seizures episodes. Despite the possible 
(a)

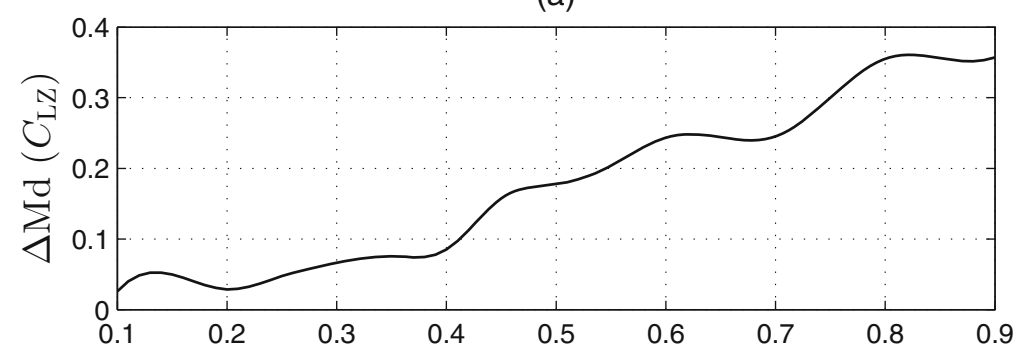

(b)

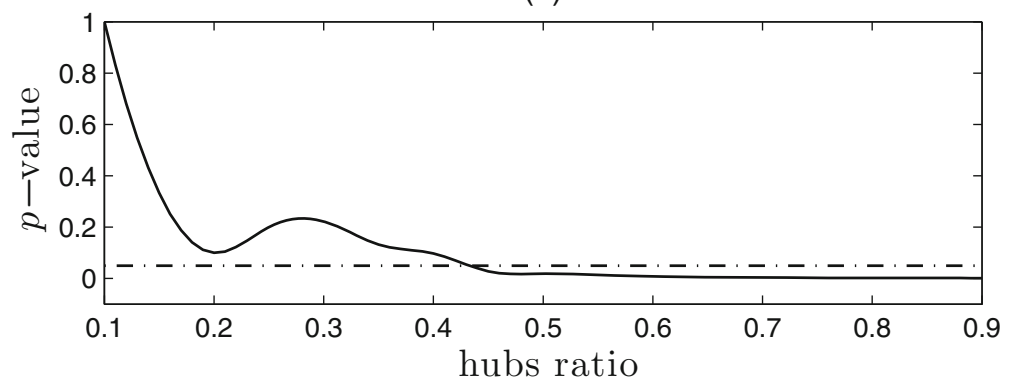

Fig. 2. (a) The difference between the median of $C_{\mathrm{LZ}}$ for networks with random and nonrandom connections. (b) $p$-value of Wilcoxon test between the $C_{\mathrm{LZ}}$ from random and non random networks. The dashed line indicates the $\alpha$ of 0.01 .

consequences of the post-ictal on the healthy brain state, few works are dedicated to the analysis of this period, an often neglected state in clinical epilepsy studies [31,32].

In order to investigate the effect of the ictal topology over the post-ictal signal, we created the hubs structures only during an interval of simulation time, from 300 to $500 \mathrm{~ms}$. After that, the adjacency matrix was restored to its values before the hubs addition. The upper part of Fig. 3 shows two LFP signals from this simulation, where before $300 \mathrm{~ms}$ both signals are equal. When the adjacency matrix is modified, the LFP signals diverge but never become exactly equal again.

This difference was approached considering a long memory process due to hubs addition. Therefore, we calculated the Hurst exponent in disjoint time windows. This exponent was persistent even after the adjacency matrix is restored by elimination of the hubs, which shows the effect of the perturbation of the ictal into post-ictal signal. The bottom part of the Fig. 3 shows the boxplot of the $H$ for each time window for networks with hubs (left) and random (right). The LFP from random networks shows $H<0.5$ for all time windows, whereas the network with hubs present a $H>0.5$ in the interval with hubs. This may imply that the synchronization of the network has a long term effect.

Moreover, the time window 6 , immediately after the restoration of the previous adjacency matrix, also shows a $H>0.5$ for more than half of the LFP simulations. This behavior resembles the post-ictal state, when the synchronism between brain regions remains higher for a brief period, even after the end of the seizure.

\section{Conclusions}

Summarizing, using the model of Izhikevich, known for its simplicity and efficiency in modelling neurons, we have studied the influence that the structure (random and 

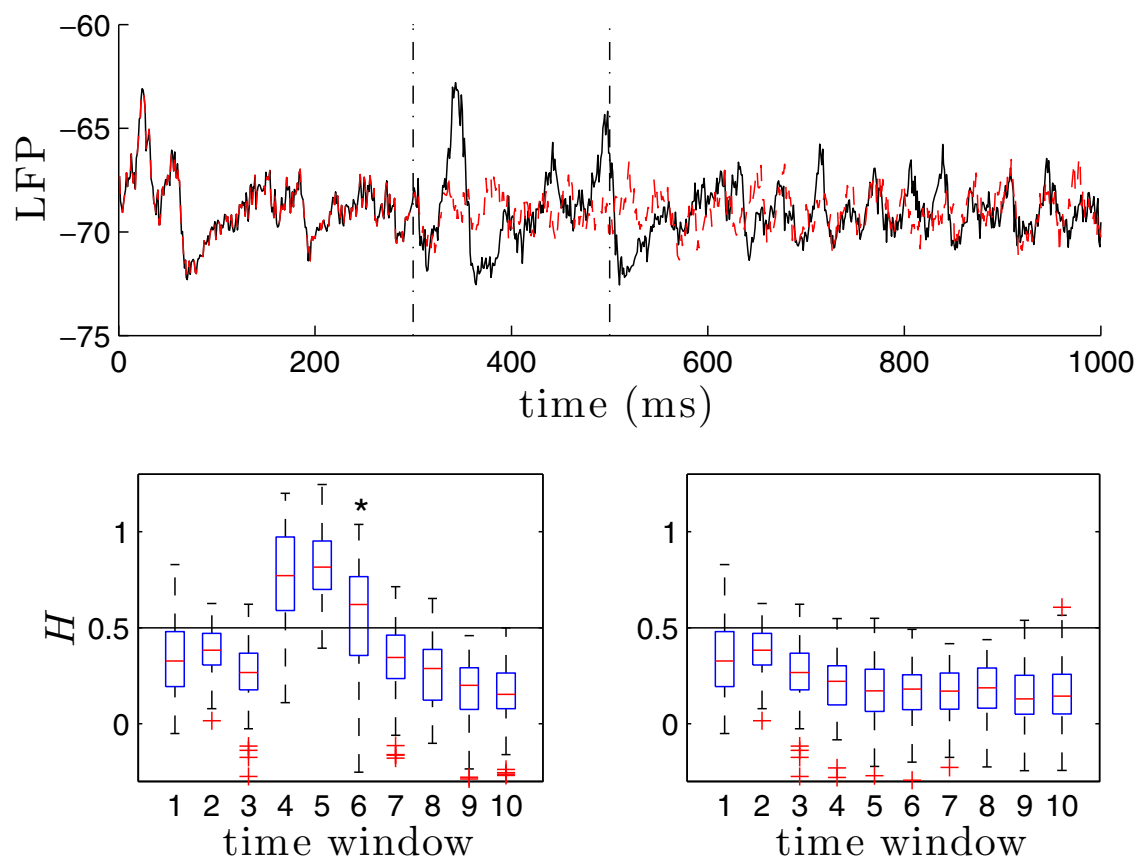

Fig. 3. Upper figure: evolution of two LFP signals along time, the black line is the network which received hubs in time $300 \mathrm{~ms}$, the red line never received hubs. The vertical dashed lines point to the interval where the hubs were present in the adjacency matrix of the black signal. Bottom left: boxplot for the Hurst exponent $H$ calculated for 10 disjoint time windows with $100 \mathrm{H}$ values. Bottom right: the same boxplot layout for the network without hubs.

non random with hubs) has in the synchronization of a network. We identify the high synchronization state with the ictal state of the brain well known in epilepsy and also study how the presence of hubs affects the post-ictal answer in the LFP.

BSM thanks the UNIEMP for their support. HAC thanks the FAPESP (process 2011/ 11973-4) for their support.

\section{References}

1. D.S. Bassett, E.T. Bullmore, Cur. Opin. Neurol. 22, 340 (2009)

2. M.E. Lynall, D.S. Bassett, R. Kerwin, P.J. McKenna, M. Kitzbichler, U. Muller, E.T. Bullmore, J. Neurosci. 30, 9477 (2010)

3. A. Fornito, A. Zalesky, C. Pantelis, E.T. Bullmore, Neuroimage 62, 2296 (2012)

4. F. Shin, L. Wang, Z. Peng, C.-Y. Wee, D. Shen, PLoS ONE 8, e63131 (2013)

5. W. de Haan, K. Mott, E.C. van Straaten, P. Scheltens, C.J. Stam, PLoS Comput. Biol. 8, e1002582 (2012)

6. M.D. Greicius, G. Srivastava, A.L. Reiss, V. Menon, PNAS 101, 4637 (2004)

7. K. Supekar, V. Menon, D. Rubin, M. Musen, M.D. Greicius, PLoS Comput. Biol. 4, e1000100 (2008)

8. S.-H. Jin, W. Jeong, C.K. Chung, Epilepsia 56, 772 (2015)

9. M. Barthelemy, A. Barrat, A. Vespignani, Dynamical processes on complex networks (Cambridge University Press, Cambridge UK, 2008)

10. Z.-X. Wu, G. Peng, W.-X. Wang, S. Chan, E. Wong, J. Stat. Mech. Theory E 2008, P05013 (2008) 
11. G. Ehrhardt, M. Marsili, F. Vega-Redondo, Int. J. Game Theory 34, 383 (2006)

12. P. Holme, B.J. Kim, C.N. Yoon, S.K. Han, Phys. Rev. E 65, 056109 (2002)

13. L. Lemieux, J. Daunizeau, M.C. Walker, Front. Syst. Neurosci. 5, 1 (2011)

14. K. Lehnertz, G. Ansmann, S. Bialonski, H. Dickten, C. Geier, S. Porz, Physica D 267, 7 (2014)

15. A.C. Rodrigues, B.S. Machado, G. Florence, A.P. Hamad, A.C. Sakamoto, A. Fujita, L.A. Baccalá, E. Amaro Jr, K. Sameshima, Eur. Phys. J. Special Topics 223, 2933 (2014)

16. C. Zhou, J. Kurths, Chaos 16, 015104 (2006)

17. C. Zhou, J. Kurths, Phys. Rev. Lett. 96, 164102 (2006)

18. J. Gómez-Gardenes, Y. Moreno, A. Arenas, Phys. Rev. Lett. 98, 034101 (2007)

19. T. Nishikawa, A.E. Motter, Y.-C. Lai, F.C. Hoppensteadt, Phys. Rev. Lett. 91, 014101 (2003)

20. E. Izhikevich, IEEE Trans. Neural. Netw. 14, 1569 (2003)

21. T. Kreuz, D. Chicharro, M. Greschner, R.G. Andrzejak, J. Neurosci. Meth. 195, 92 (2011)

22. V. Santos Filho, B.S. Machado, G. Francisco, L. Tomio, Physica A 392, 3087 (2013)

23. P. Abry, P. Flandrin, M.S. Taqqu, D. Veitch, Theory and applications of long-range dependence (Springer Science \& Business Media, Berlin, 2003), p. 527

24. W.A. Varanda, L.S. Liebovitch, J.N. Figueiroa, R.A. Nogueira, J. Theor. Biol. 206, 343 (2000)

25. F.M. Atay, T. Biyikoglu, J. Jost, Physica D 224, 35 (2006)

26. C. Geier, S. Bialonski, C.E. Elger, K. Lehnertz, Seizure 25, 160 (2015)

27. S. Kalitzin, D. Velis, P. Suffczynski, J. Parra, F. Lopes da Silva, Clin. Neurophysiol. 116, $718(2005)$

28. H. Jing, J. Gao, J.C. Principe, IEEE Trans. Biomed. Eng. 20, 1 (2006)

29. C.J. Stam, Clin. Neurophysiol. 116, 2266 (2005)

30. M.A. Kramer, U.T. Eden, E.D. Kolaczyk, R. Zepeda, E.N. Eskandar, S.S. Cash, J. Neurosci. 30, 10076 (2010)

31. R.S. Fisher, S.C. Schachter, Epilepsy. Behav. 1, 52 (2000)

32. W.H. Theodore, Epilepsy. Behav. 19, 118 (2010) 